Open Access

\title{
Serum metabolites may be useful markers to assess vascular invasion and identify normal alpha-fetoprotein in hepatocellular carcinoma undergoing liver resection: a pilot study
}

Chao-Wei Lee ${ }^{1,2,3}$ (D), Ming-Chin Yu 1,2,3, Gigin Lin ${ }^{4,5,6}$, Jo-Chu Chiu², Meng-Han Chiang ${ }^{4}$, Chang-Mu Sung 2,3,7, Yi-Chung Hsieh ${ }^{3,7}$, Tony Kuo ${ }^{3,7}$, Cheng-Yu Lin ${ }^{3,7}$ and Hsin-I Tsai ${ }^{2,3,8^{*}}$

\begin{abstract}
Purpose: Hepatocellular carcinoma (HCC) is the most common primary malignancy of the liver with a dismal prognosis. Vascular invasion, among others, is the most robust indicator of postoperative recurrence and overall survival after liver resection for HCC. Few studies to date have attempted to search for effective markers to predict vascular invasion before the operation. The current study would examine the plasma metabolic profiling via ${ }^{1} \mathrm{H}$ NMR of HCC patients undergoing liver resection and aim to search for potential biomarkers in the early detection of HCC with normal alpha-fetoprotein (AFP) and the diagnosis of vascular invasion preoperatively.

Materials and methods: HCC patients scheduled to receive liver resections for their HCC were recruited and divided into two separate groups, investigation cohort and validation cohort. Their preoperative blood samples were collected and subjected to a comprehensive metabolomic profiling using ${ }^{1} \mathrm{H}$-nuclear magnetic resonance spectroscopy (NMR).

Results: There were 35 HCC patients in the investigation group and 22 patients in the validation group. Chronic hepatitis B remained the most common etiology of HCC, followed by chronic HCV infection. The two study cohorts were essentially comparable in terms of major clinicopathological variables. After ${ }^{1} \mathrm{H}$-nuclear NMR analysis, we found in the investigation cohort that HCC with normal alpha-fetoprotein (AFP $<15 \mathrm{ng} / \mathrm{mL}$ ) had significantly higher serum level of O-acetylcarnitine than those with higher AFP (AFP $\geq 15 \mathrm{ng} / \mathrm{mL}, P=0.025$ ). In addition, HCC with microscopic vascular invasion $(\mathrm{VI})$ had significantly higher preoperative serum level of formate than HCC without microscopic VI $(P=0.023)$. These findings were similar in the validation cohort.

(Continued on next page)
\end{abstract}

\footnotetext{
* Correspondence: tsaic@hotmail.com

${ }^{2}$ Graduate Institute of Clinical Medical Sciences, Chang Gung University,

Taoyuan, Taiwan

${ }^{3}$ College of Medicine, Chang Gung University, Taoyuan, Taiwan

Full list of author information is available at the end of the article
}

(c) The Author(s). 2020 Open Access This article is licensed under a Creative Commons Attribution 4.0 International License, which permits use, sharing, adaptation, distribution and reproduction in any medium or format, as long as you give appropriate credit to the original author(s) and the source, provide a link to the Creative Commons licence, and indicate if changes were made. The images or other third party material in this article are included in the article's Creative Commons licence, unless indicated otherwise in a credit line to the material. If material is not included in the article's Creative Commons licence and your intended use is not permitted by statutory regulation or exceeds the permitted use, you will need to obtain permission directly from the copyright holder. To view a copy of this licence, visit http://creativecommons.org/licenses/by/4.0/ The Creative Commons Public Domain Dedication waiver (http://creativecommons.org/publicdomain/zero/1.0/) applies to the data made available in this article, unless otherwise stated in a credit line to the data. 
(Continued from previous page)

Conclusion: A comprehensive metabolomic profiling of HCC demonstrated that serum metabolites may be utilized to assist the early diagnosis of AFP-negative HCC patients and recognition of microvascular invasion in order to facilitate preoperative surgical planning and postoperative follow-up. Further, larger scale prospective studies are warranted to consolidate our findings.

Keywords: Metabolomics, Metabolites, Vascular invasion, Formate, Hepatocellular carcinoma, Hepatoma

\section{Introduction}

Hepatocellular carcinoma (HCC) is the most common primary malignancy of the liver with an estimated incidence of mortality at 700,000 per year worldwide [1]. In Taiwan, HCC is the second most common cause of cancer death, with more than 8000 deaths annually [2]. Surgical resections remain the most effective therapy in selected patients; however, the extent and feasibility of such are still complicated by the patients' coexisting liver diseases, such as chronic hepatitis $\mathrm{B}$ or $\mathrm{C}$ and alcoholic liver disease. Less than $40 \%$ of non-cirrhotic HCC patients received liver resections, and the rate was even much lower in patients with chronic liver disease or overt cirrhosis [3]. Regardless a significant improvement in the surveillance program, diagnostic modality, surgical techniques, and postoperative care, the recurrence rate of HCC after liver resection, liver transplantation, or radiofrequency ablation (RFA) still remains as high as $60 \%$ [4]. Given the low operative and high recurrence rate of $\mathrm{HCC}$, it is therefore of paramount importance to search for potential markers in the prediction of tumor recurrence or long-term survival after liver resection to ensure optimal surveillance or opportune adjuvant treatment postoperatively.

Risk factors for HCC recurrence after surgery have been identified, including lymph node metastasis (LNM) [5], vascular invasion, poor tumor differentiation, large tumor size, and multinodularity [6, 7], among which, vascular invasion $(\mathrm{VI})$, being the most robust indicator of postoperative recurrence and overall survival [8]. VI can be subdivided into macroscopic VI when larger or major vessels are invaded and detectable by preoperative radiologic study and microscopic VI when small blood vessels are invaded and identified only under microscopic examination. In the presence of preoperative VI, more aggressive surgical treatment such as wider safety margin or anatomical liver resection can be performed. Moreover, when VI is highly suspected, perioperative transarterial chemoembolization (TACE) can be applied. That said, microscopic VI is barely detectable by conventional radiologic studies. Few studies to date have attempted to search for effective markers in the prediction of preoperative microscopic VI. An overexpression of abnormal spindle-like microcephaly associated (ASPM) and upregulation of cytohesin-3 have been found to be associated with VI and early tumor recurrence $[9,10]$. Nevertheless, these molecular markers are barely detectable in percutaneous biopsy or before the operation. For real-world clinical practicing, a reliable predictive marker readily available from blood samples may be the best resort.

Metabolomics, a new science that simultaneously detects many metabolites in biofluids and tissues, has been employed in the fields of oncology and various other diseases for the evaluation of their altered levels in a pathological state. Recently, metabolomics has also been employed in the field of HCC in search for diagnostic as well as predictive biomarkers for HCC. HCC patients in different pathological status were found to possess different plasma profiling [11]. For example, Di Poto et al. had demonstrated that a panel consisting of 11 serum metabolites and three clinical factors (AFP, Child-Pugh score, and etiologic factors) had a higher area under the ROC curve compared to AFP alone for the diagnosis of HCC [12, 13]. Luo et al. identified a biomarker panel (phenylalanyl-tryptophan and glycocholate) that had a better diagnostic value compared to AFP for distinguishing HCC patients from a high-risk cohort of cirrhotic patients [14]. Pathways of lysophospholipid, sphingolipid, bile acid, amino acid, and reactive oxygen species metabolism were found to be consistently associated with the development of HCC [15]. Moreover, increased uptake of glucose, enhanced lactate production, and altered lipid metabolism have been defined to be characteristic metabolic signatures in HCC [16]. As for the prediction of outcome, by using metabolomic platforms, ethanolamine, lactic acid, acotinic acid, phenylalanine, and ribose were found useful in discriminating patients with early recurrence from those without recurrence [17], and metabolomic fingerprints rather than tumor size appeared to show an impact on poor prognosis [18]. Lately, Zhou et al. [19] identified that a combination of serum methionine, GCDCA, and cholesterol sulfate levels could predict early HCC recurrence with high accuracy. Despite these remarkable results, few studies to date have tried to search for potential metabolites predictive of microscopic VI in HCC. In this study, we present plasma metabolic profiling via ${ }^{1} \mathrm{H}$-nuclear magnetic resonance spectroscopy ( $\left.{ }^{1} \mathrm{H}-\mathrm{NMR}\right)$ of $\mathrm{HCC}$ patients undergoing liver resection and aim to search for 
potential biomarkers in the early detection of $\mathrm{HCC}$ with normal alpha-fetoprotein (AFP) and the diagnosis of VI preoperatively.

\section{Materials and methods Patient selection}

Under the approval of the Institutional Review Boards of Chang Gung Memorial Hospital (CGMH), primary HCC patients scheduled to receive curative liver resection for their tumors were included into our study. After informed consent was completed, $10 \mathrm{ml}$ of blood was drawn both before the operation and 1 month after the operation. The patients were asked to stay fasted prior to blood tests. The serum was extracted by centrifugation and was stored into aliquots and frozen at $-80^{\circ} \mathrm{C}$ until further batch analysis by hydrogen- 1 nuclear magnetic resonance $\left({ }^{1} \mathrm{H}-\mathrm{NMR}\right)$ in the Metabolomic Core Laboratory at CGMH and Chang Gung University (CGU). Patient demographic data including age, gender, cigarette smoking, alcohol consumption, hepatitis B virus (HBV) infection, anti-hepatitis C virus antibody (anti-HCV) level, serum bilirubin, albumin, prothrombin levels, international normalized ratio (INR), serum alanine aminotransferase (ALT) activity, serum aspartate aminotransferase (AST) activity, serum gammaglutamyltransferase (GGT) activity, platelet count, and alpha-fetal protein (AFP) level were collected. Their pathological factors and oncological outcome including tumor lymph node metastasis status, tumor encapsulation, histological grade, VI, daughter nodules, resection margin, fatty change, liver cirrhosis, tumor recurrence, and longterm survival were also recorded. Early recurrence is defined as the occurrence of tumor recurrence within 2 years of the curative operation. Overall survival (OS) is defined by the time elapsing from the date of diagnosis to either the date of death or the date of the last contact. Cases with surgical mortality, defined as death within 1 month of surgery were excluded from the survival analyses. A separate cohort was also recruited for validation.

\section{NMR spectroscopy analysis General procedure}

The procedures briefly described below were aimed to obtain high-quality information for metabolic study, including (a) chemical shifts (value, TMS, or TSP as 0.0), (b) spin-spin coupling and coupling constants (J value), (c) relaxation (may not necessary all times), and (d) signal intensity (for quantitation). The standardized procedure currently used in the Metabolomic Core Laboratory, CGU, is a modified protocol of that described by Beckonert et al. [20].

\section{NMR measurement}

Measurement of the ${ }^{1} \mathrm{H}$-NMR spectra for aqueous extracts A NOESY pulse sequence with water irradiation during the relaxation delay and during the mixing time

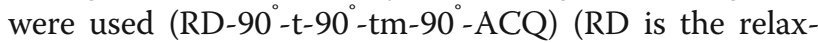
ation delay, $t$ is a short delay typically of about $3 \mathrm{~s}, 90^{\circ}$ represents a $90^{\circ} \mathrm{RF}$ pulse, tm is the mixing time, and ACQ was the data acquisition period. The mixing time is $\mathrm{tm}=100-150 \mathrm{~ms})$. Other parameters are as follows: spectral width $=20 \mathrm{ppm}$, number of time domain data points $=32,768$, relaxation delay, $\mathrm{RD}=2.0 \mathrm{~s}$, acquisition time $=1.36 \mathrm{~s}$, number of scans $=64$ for urine and 128 for plasma, serum, and tissue extracts. This resulted in a total acquisition time of about $8-12$ min per sample (128 scans) [20]. The pulse sequence was suitable for NMR signal quantitation.

Lipophilic metabolites (lipidomics) The ${ }^{1} \mathrm{H}-\mathrm{NMR}$ spectra of lipid extracts did not require water suppression. A routine $90^{\circ}$ pulse sequence was sufficient in measuring fully relaxed spectra (for quantitation purpose). In this core laboratory, a routine collect 64 transients (NS) into 32,768 data points was carried out. A relaxation delay $=2 \mathrm{~s}$, spectral width $=20 \mathrm{ppm}$, and acquisition time $=1.36 \mathrm{~s}$ can be used [20]. For quantitation, signal intensity was used. A mixture of lipid metabolites containing known amounts of free cholesterol and cholesterol oleate, phospholipids, and TG were used as reference sample. The crude lipid extracts (Folch method) of a mixture of plasma samples were used for calibration.

NMR data management The conversion of FID signals to spectral data was carried out using the softwares of the available software package of NMR instruments. Assignment of the chemical shifts to specific metabolites will base on spectral data in the literature or by the information based on the measurement of authentic metabolites in our chemical library. An NMR data base (not complete) of LMW metabolites (pH adjusted) was available. Several non-commercial and commercial application softwares such as MetaboAnalyst [21] were used for metabolite identification. The NMR spectrum was normalized by internal standard (TSP, 3-(Trimethylsilyl)propionic-2,2,3,3-d4 acid sodium salt). The missing value of the identified metabolites was defined as zero.

\section{Statistical analysis}

The statistical analysis was performed with IBM SPSS Statistics 21 (IBM Corporation, Software Group, Somers, NY, USA). Fisher's exact test and Pearson's $\mathrm{X} 2$ test were used to analyze categorical data. Student's $t$ test was used to analyze quantitative variables. Statistical significance is defined as $P<0.05$. 
Table 1 Patient demographics and tumor characteristics of the investigation cohort $(n=35)$

\begin{tabular}{|c|c|}
\hline & No (\%) \\
\hline \multicolumn{2}{|c|}{ Age (year) (mean $62.7 \pm 1.9)$} \\
\hline$\leqq 65$ & $20(57.1)$ \\
\hline$>65$ & $15(42.9)$ \\
\hline \multicolumn{2}{|l|}{ Gender } \\
\hline Male & $27(77.1)$ \\
\hline Female & $8(22.9)$ \\
\hline \multicolumn{2}{|l|}{ Smoking } \\
\hline Yes & $11(31.4)$ \\
\hline No & $24(68.6)$ \\
\hline \multicolumn{2}{|l|}{ Alcohol } \\
\hline Yes & $6(17.1)$ \\
\hline No & $29(82.9)$ \\
\hline \multicolumn{2}{|l|}{ HBsAg } \\
\hline Positive & $23(65.7)$ \\
\hline Negative & $12(34.3)$ \\
\hline \multicolumn{2}{|l|}{ Anti-HCV Ab } \\
\hline Positive & $8(22.9)$ \\
\hline Negative & $27(77.1)$ \\
\hline \multicolumn{2}{|l|}{ Cirrhosis } \\
\hline Yes & $14(40.0)$ \\
\hline No & $21(60.0)$ \\
\hline \multicolumn{2}{|l|}{ ICG-15 (\%) } \\
\hline$\leqq 10$ & $20(57.1)$ \\
\hline$>10$ & $15(42.9)$ \\
\hline \multicolumn{2}{|l|}{ AFP (ng/ml) } \\
\hline$\leqq 15$ & $14(40.0)$ \\
\hline$>15$ & $21(60.0)$ \\
\hline \multicolumn{2}{|l|}{ AFP (ng/ml) } \\
\hline$\leqq 200$ & $27(77.1)$ \\
\hline$>200$ & $8(22.9)$ \\
\hline \multicolumn{2}{|c|}{ Surgical procedure } \\
\hline Major & $9(25.7)$ \\
\hline Minor & $26(74.3)$ \\
\hline \multicolumn{2}{|l|}{ Size $(\mathrm{cm})$} \\
\hline$\leqq 5$ & $24(68.6)$ \\
\hline$>5$ & $11(31.4)$ \\
\hline \multicolumn{2}{|c|}{ Encapsulation } \\
\hline Yes & $28(80.0)$ \\
\hline No & $7(20.0)$ \\
\hline \multicolumn{2}{|c|}{ Vascular invasion } \\
\hline Yes & $14(40.0)$ \\
\hline No & $21(60.0)$ \\
\hline
\end{tabular}

Tumor rupture
Table 1 Patient demographics and tumor characteristics of the investigation cohort $(n=35)$ (Continued)

\begin{tabular}{ll}
\hline & No (\%) \\
\hline Yes & $3(8.6)$ \\
No & $32(91.4)$ \\
Daughter nodules & \\
Yes & $4(11.4)$ \\
No & $31(88.6)$ \\
Resection margin & \\
Positive & $0(0)$ \\
Negative & $35(100)$ \\
Edmondson grade & \\
I/II & $4(11.8) / 10(28.6)$ \\
III/IV & $18(52.9) / 3(8.8)$ \\
Early recurrence & \\
Yes & \\
No & $4(11.4)$ \\
\hline aTumor recurren & $31(88.6)$ \\
\hline
\end{tabular}

${ }^{a}$ Tumor recurrence within 2 years after surgery

\section{Results}

Patient characteristics

A total of 35 patients were enrolled to the study as the investigation group. Their blood samples were collected the day before and 1 month after the operation. As shown in Table 1, of the 35 patients in the investigation cohort, the majority were males, with an average of 62.7 \pm 1.9 years of age. Twenty-three patients $(65.7 \%)$ had HBV infection and 8 (22.9\%) had HCV infection. The majority of the investigation group had serum AFP levels greater than $15 \mathrm{ng} / \mathrm{mL}$, with an average of $16,096 \pm 12$, $568 \mathrm{ng} / \mathrm{mL}$ (Table 2). Nine patients received major surgical procedures, and about $70 \%$ of tumors were less than $5 \mathrm{~cm}$ in diameter. Among the investigation group, 20\% was non-encapsulated HCC, $40 \%$ had VI, $11.4 \%$ had daughter nodules, $8.6 \%$ was ruptured tumor, and $40 \%$ had pathologically proven cirrhosis. Using Edmondson and Steiner grade, the distribution of grade I to IV HCC in the investigation cohort was $11.8 \%, 28.6 \%, 52.9 \%$, and $8.8 \%$, respectively. Another 22 patients were recruited as the validation cohort with similar clinicopathological characteristics as the investigation cohort, except all 22 patients in the validation cohort were encapsulated HCC (Table 2).

\section{NMR plasma profiles in HCC patients in relation to serum AFP levels}

As demonstrated in Fig. 1, heatmap showed the clustering of metabolites that highly correlated with the status of HCC in the absence of AFP elevation. Also, ${ }^{1} \mathrm{H}-\mathrm{NMR}$ plasma profiles using OPLS-DA, in which red area denoted HCC patients with normal AFP levels (less than $15 \mathrm{ng} / \mathrm{mL}$ ) while green area denoted 
Table 2 Comparison of clinicopathological characteristics between investigation cohort $(n=35)$ and validation cohort ( $n=22)$

\begin{tabular}{|c|c|c|c|c|c|c|c|}
\hline & \multicolumn{3}{|c|}{ Categorical variables $(\boldsymbol{n}(\%))$} & & \multicolumn{3}{|c|}{ Continuous variables (mean $\pm \mathrm{SE}$ ) } \\
\hline & Investigation & Validation & $\boldsymbol{P}$ value & & Investigation & Validation & $\boldsymbol{P}$ value \\
\hline Male gender & $27(77.1)$ & $19(86.4)$ & 0.502 & Age (year) & $62.7 \pm 1.9$ & $60.2 \pm 2.4$ & 0.429 \\
\hline HBV infection & $23(65.7)$ & $11(50.0)$ & 0.277 & BMI $\left(\mathrm{Kg} / \mathrm{m}^{2}\right)$ & $23.9 \pm 0.5$ & $25.6 \pm 0.7$ & 0.063 \\
\hline HCV infection & $8(22.9)$ & $9(40.9)$ & 0.234 & ICG-15 (\%) & $9.53 \pm 0.88$ & $11.44 \pm 1.64$ & 0.316 \\
\hline AFP > $15 \mathrm{ng} / \mathrm{mL}$ & $21(60.0)$ & $10(45.5)$ & 0.413 & AFP (ng/mL) & $16096 \pm 12568$ & $17938 \pm 17777$ & 0.931 \\
\hline Major procedure & $9(25.7)$ & $6(27.3)$ & 1.000 & CEA (ng/mL) & $6.07 \pm 4.07$ & $1.57 \pm 0.20$ & 0.361 \\
\hline Encapsulation & $28(80.0)$ & $22(100.0)$ & 0.036 & CA $19-9(\mathrm{U} / \mathrm{mL})$ & $26.6 \pm 3.7$ & $22.1 \pm 3.9$ & 0.421 \\
\hline Vascular invasion & $14(40.0)$ & $10(45.5)$ & 0.785 & Size $(\mathrm{cm})$ & $4.59 \pm 0.61$ & $4.05 \pm 0.68$ & 0.557 \\
\hline Daughter nodules & $4(11.4)$ & $2(9.1)$ & 1.000 & & & & \\
\hline Tumor rupture & $3(8.6)$ & $1(4.5)$ & 1.000 & & & & \\
\hline Cirrhosis & $14(40.0)$ & $13(59.1)$ & 0.184 & & & & \\
\hline $\begin{array}{l}\text { Edmondson and Steiner Grade } \\
(\mathrm{I} / \mathrm{II} / \mathrm{III} / \mathrm{IV})\end{array}$ & $\begin{array}{l}4(11.8) / 10(28.6) / \\
18(52.9) / 3(8.8)\end{array}$ & $\begin{array}{l}3(13.6) / 13(59.1) / \\
5(22.7) / 1(4.5)\end{array}$ & 0.073 & & & & \\
\hline
\end{tabular}

HCC patients with abnormal AFP levels (greater or equal to $15 \mathrm{ng} / \mathrm{mL}$ ), have shown a separation between the patients with normal $(<15 \mathrm{ng} / \mathrm{mL})$ and elevated AFP $(\geq 15 \mathrm{ng} / \mathrm{mL})$.

Further analysis on the identifications of metabolites associated with AFP levels revealed a few metabolites possibly responsible for the group separation, including glycine, O-acetylcarnitine, histidine, and ornithine. Of particular interest, the level of $\mathrm{O}$-acetylcarnitine was much higher in the normal AFP-HCC group (N-HCC) than abnormal AFP-HCC group (A-HCC) with statistical significance $(0.600 \pm 0.08$ vs. $0.374 \pm 0.11, P$ value

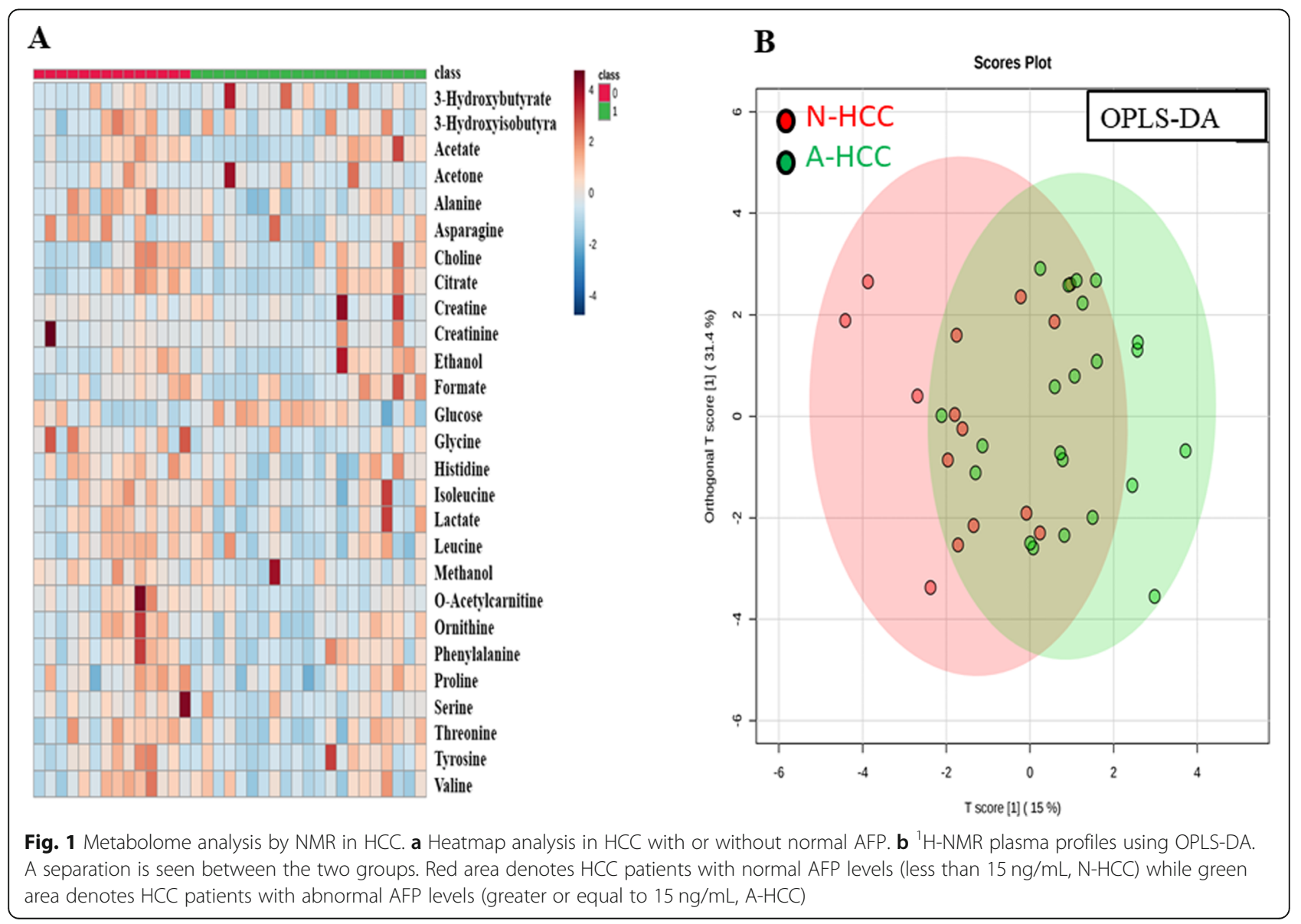


Table 3 Nuclear magnetic resonance (NMR) observed serum metabolites with intensity differences associated with different AFP for hepatocellular carcinoma

\begin{tabular}{llll}
\hline Metabolites & AFP $<15 \mathrm{ng} / \mathrm{ml}$ & AFP $\geq 15 \mathrm{ng} / \mathrm{ml}$ & $\boldsymbol{P}$ value \\
\hline Investigation cohort $(\boldsymbol{n}=\mathbf{3 5})$ & & \\
Glycine & $26.128 \pm 2.83$ & $18.627 \pm 1.09$ & 0.006 \\
O-Acetylcarnitine & $0.600 \pm 0.08$ & $0.374 \pm 0.11$ & 0.025 \\
Histidine & $6.597 \pm 0.36$ & $5.532 \pm 0.37$ & 0.062 \\
Ornithine & $9.263 \pm 1.05$ & $7.065 \pm 0.58$ & 0.073 \\
Validation cohort $(\boldsymbol{n}=\mathbf{2 2})$ & & \\
Glycine & $26.810 \pm 1.33$ & $27.195 \pm 2.19$ & 0.752 \\
O-Acetylcarnitine & $0.958 \pm 0.09$ & $0.687 \pm 0.08$ & 0.032 \\
Histidine & $7.303 \pm 0.32$ & $6.070 \pm 0.55$ & 0.153 \\
Ornithine & $10.504 \pm 0.86$ & $9.061 \pm 0.75$ & 0.215 \\
\hline
\end{tabular}

Metabolites were expressed as mean \pm SE in arbitrary units ${ }^{\text {a }}$ Student's $t$ test

0.025). Similar trend was observed in the validation group in which the level of $\mathrm{O}$-acetylcarnitine was 0.958 \pm 0.09 vs. $0.687 \pm 0.08$ in $\mathrm{N}-\mathrm{HCC}$ and $\mathrm{A}-\mathrm{HCC}$, respectively, as shown in Table 3.

The diagnostic ability of $\mathrm{O}$-acetylcarnitine in discriminating $\mathrm{N}-\mathrm{HCC}$ from the entire $\mathrm{HCC}$ cohort was demonstrated by the ROC curves in Fig. 2. In terms of N-HCC,
O-acetylcarnitine has shown an area under the curve (AUC) of the receiver-operating characteristics (ROC) or AUROC of 0.753 in the investigation group and an AUROC of 0.718 in the validation group.

\section{NMR plasma profiles in HCC patients in relation to VI}

As VI is one important prognostic factor in HCC and can potentially alter the extent of surgical treatment, ${ }^{1} \mathrm{H}-\mathrm{NMR}$ was conducted in an attempt to distinguish the status of microvascular invasion in HCC patients preoperatively. In Fig. 3, heatmap and OPLS-DA demonstrated a separation of metabolites contributing to the microvascular invasion. Red and green denoted the absence and the presence of microvascular invasion. From this model, few metabolites with high variable importance projection (VIP) scores were selected. In Fig. 4, formate appeared to contribute to the separation with the highest VIP score [22]. The level of formate was significantly higher in HCC with VI than HCC without VI (37.592 \pm 7.83 vs. $19.697 \pm$ $3.23, P$ value 0.023 ) (Table 4 ). Similar trend was observed in the validation group in which the level of formate was $18.776 \pm 0.99$ vs. $14.807 \pm 1.48$ in $\mathrm{HCC}$ with and without VI, respectively.

\section{Investigation cohort AUROC 0.753}

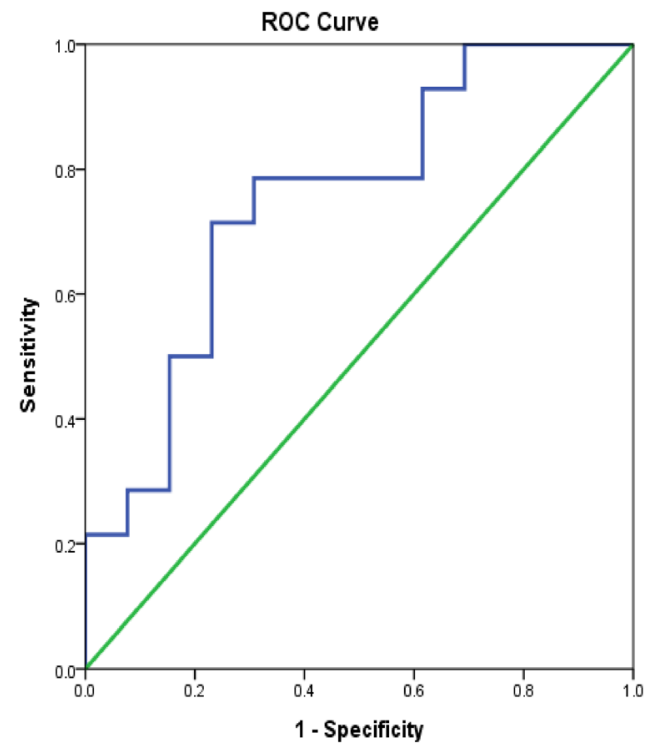

\section{Validation cohort AUROC 0.718}

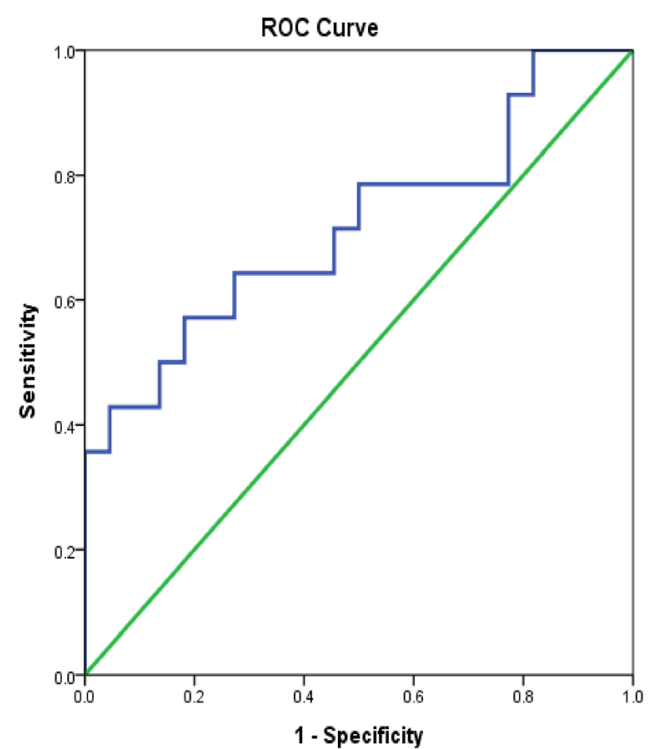

Fig. 2 ROC curves of O-acetylcarnitine for HCC with normal AFP. In the entire HCC group, O-acetylcarnitine has shown an area under the curve (AUC) of the receiver-operating characteristics (ROC) or AUROC of 0.753 in the investigation group and an AUROC of 0.718 in the validation group for N-HCC 


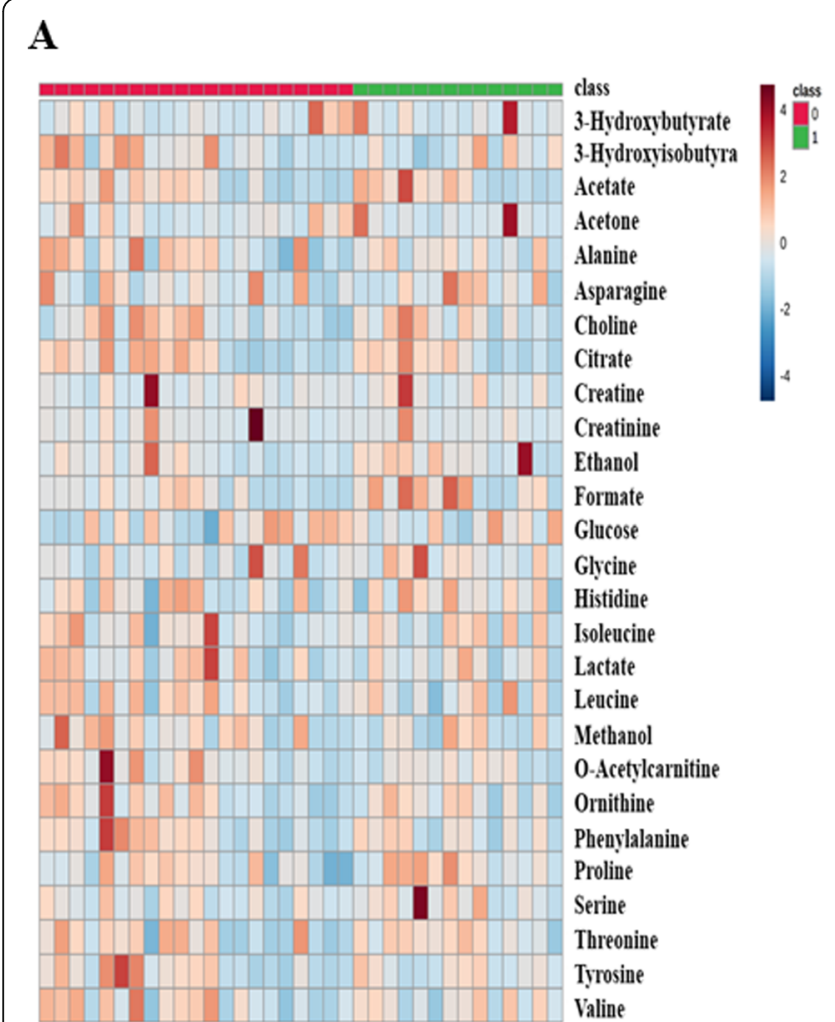

B

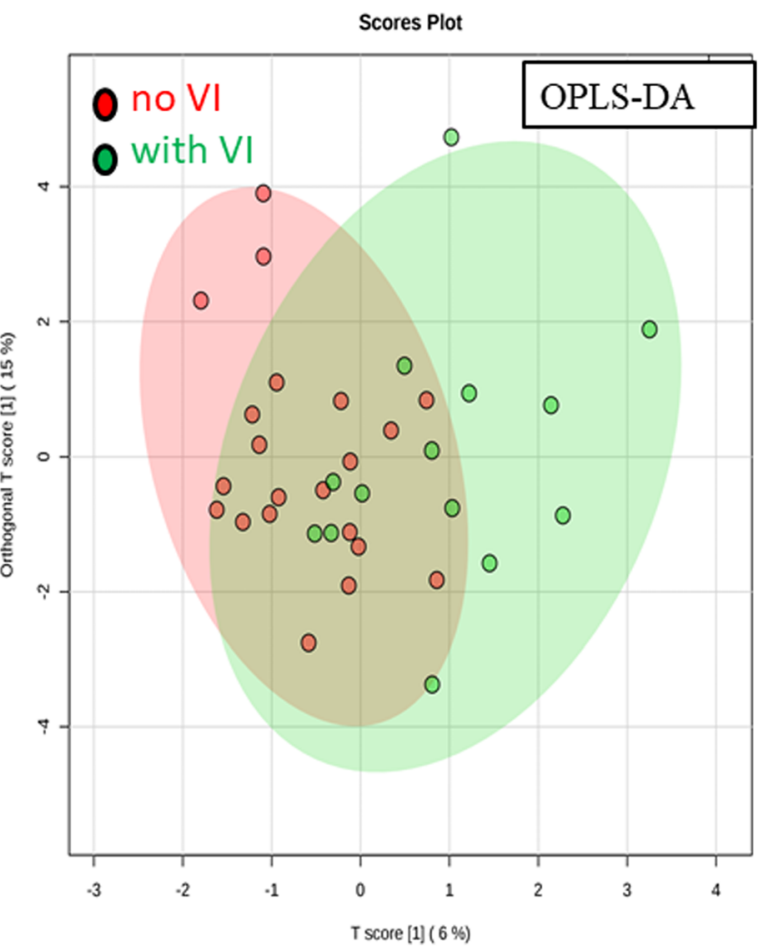

Fig. 3 Metabolome analysis by NMR in HCC in association with vascular invasion. a Heatmap analysis with or without microscopic vascular invasion. $\mathbf{b}^{1} \mathrm{H}$-NMR plasma profiles using OPLS-DA. A separation is seen between the two groups. Red area denotes HCC patients without vascular invasion while green area denotes HCC patients with vascular invasion

\section{Discriminative ability of clinical parameters and biomarker for VI}

As previously demonstrated, formate was highly selective for the presence of microvascular invasion in HCC patients. Tumor size, serum AFP, and ICG-15 were compared between HCC patients with and without microscopic VI. As shown in Table 4 and Fig. 5, even though tumor size, AFP, and ICG-15 individually may not be adequate in distinguishing the presence of VI, formate appeared to display an acceptable AUROC of 0.612 in the investigation group and 0.727 in the validation group. In combination of formate, AFP, and tumor size, the AUROC in predicting microscopic VI raised to 0.806 in the investigation group and 0.745 in the validation group (Fig. 6).

\section{Discussions}

HCC is one of the most common malignant tumors, with increasing incidence and high mortality rate, in the world [23, 24]. Clinically, HCC is diagnosed by tissue-based histopathological findings, characteristic radiologic features, or blood-based assays such as AFP, and a majority of $\mathrm{HCC}$ are diagnosed at an advanced stage. It appears that the sensitivity and specificity of such clinically used methodology may not be adequate in identifying early stage HCC and thus significantly reducing the reliability. Recently, metabolomics has gained its popularity in the field of surgical oncology as homeostasis perturbations related to cancer development may be detected with the new platform. As an extreme complex organ responsible for glycolysis and metabolisms of carbohydrates, amino acids, and fatty acids, literature has revealed that metabolomics based on NMR could be used to identify biomarkers in varying stages of liver diseases [25] in hope for early tumor detection.

AFP level is the most frequently used tumor marker for HCC; however, in the absence of AFP elevation, the diagnosis can be difficult. Metabolomic analysis comparing $\mathrm{HCC}$ and healthy individuals has demonstrated that an increased level of acetylcarnitine is associated with HCC and highly correlated with tumor grade [26-28]. In consistency with the literature, we also found elevated serum acetylcarnitine, more so in these normal AFP HCC patients than those with abnormal AFP. Among HCC patients, acetylcarnitine showed a discriminative accuracy of $>70 \%$ for those 


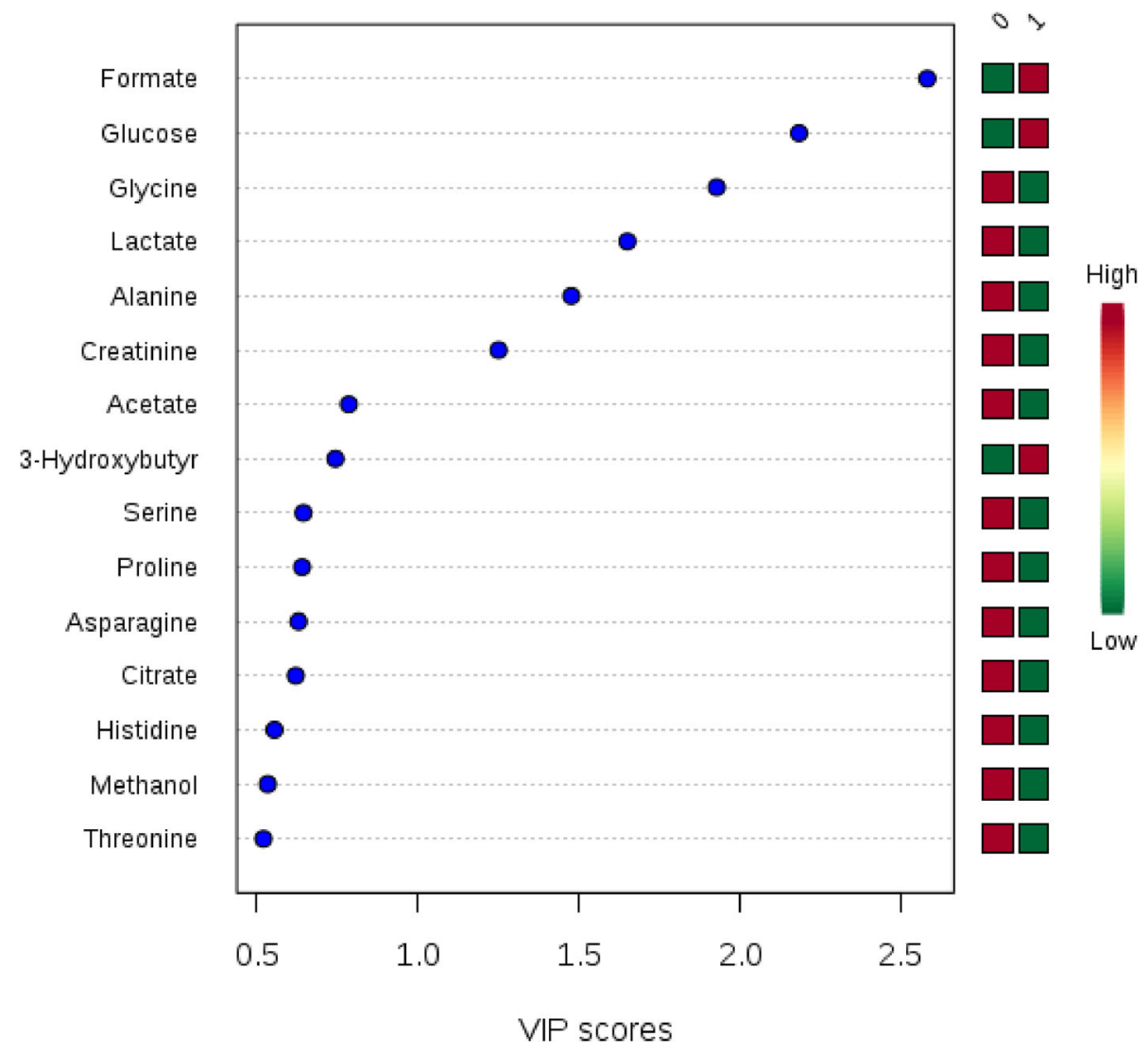

Fig. 4 PLS-DA variable importance projection (VIP) scores. The metabolites responsible for the classification of VI or not would be identified using the variable importance in projection (VIP) scores. Metabolites with high VIP are more important in providing class separation, while those with small VIP provide less contribution [22]. Formate had the highest VIP score in terms of VI. Red or green on the right of Fig. 4 indicated the low or high concentration of metabolites by comparing the concentration of each metabolite in patients with (1) or without (0) vascular invasion

Table 4 Clinical variables and nuclear magnetic resonance (NMR) observed serum metabolites with intensity differences associated with vascular invasion (VI) for hepatocellular carcinoma

\begin{tabular}{|c|c|c|c|}
\hline Metabolites & Without VI & With Vl & $\boldsymbol{P}$ value $^{\text {a }}$ \\
\hline \multicolumn{4}{|c|}{ Investigation cohort $(n=35)$} \\
\hline Tumor size $(\mathrm{cm})$ & $3.47 \pm 0.49$ & $6.29 \pm 1.22$ & 0.047 \\
\hline $\operatorname{AFP}(\mathrm{ng} / \mathrm{ml})$ & $6541.91 \pm 6486.76$ & $30428.30 \pm 30156.35$ & 0.360 \\
\hline ICG-15 (\%) & $9.80 \pm 1.10$ & $9.11 \pm 1.54$ & 0.711 \\
\hline Formate & $19.697 \pm 3.23$ & $37.592 \pm 7.83$ & 0.023 \\
\hline \multicolumn{4}{|c|}{ Validation cohort $(n=22)$} \\
\hline Tumor size $(\mathrm{cm})$ & $3.80 \pm 0.98$ & $4.33 \pm 0.95$ & 0.710 \\
\hline $\operatorname{AFP}(\mathrm{ng} / \mathrm{ml})$ & $145.82 \pm 87.38$ & $39289.31 \pm 39109.13$ & 0.343 \\
\hline ICG-15 (\%) & $13.18 \pm 2.42$ & $9.35 \pm 2.10$ & 0.256 \\
\hline Formate ${ }^{\mathrm{b}}$ & $14.807 \pm 1.48$ & $18.776 \pm 0.99$ & 0.042 \\
\hline
\end{tabular}

Data were expressed as mean \pm SE

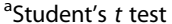

${ }^{\mathrm{b}}$ Expressed in arbitrary units with normal AFP (AFP $<15 \mathrm{mg} / \mathrm{L}$ ), suggesting that acetylcarnitine may be served as a sensitive and specific biomarker, in complementary to AFP, for monitoring the development of HCC. Acetylcarnitine is an acetylated form of L-carnitine mainly used for energy production by transporting activated long chain fatty acids from the cytosol into mitochondria. Carnitine is naturally produced by the body. As advanced stage of HCC often predisposes patients to cancer cachexia, low serum levels of acetylcarnitine are mainly the result of reduced synthesis of carnitine in HCC patients. Further studies are warranted to elucidate the roles of acetylcarnitine in the diagnosis and follow-up of HCC patients.

In clinical practice, the presence of VI may influence the surgeons' decision in terms of the extent of resection. VI is found to be associated with the presence of ascites in patients with HBV-related cirrhotic HCC [29]. Other evidence has also shown that derived neutrophil 


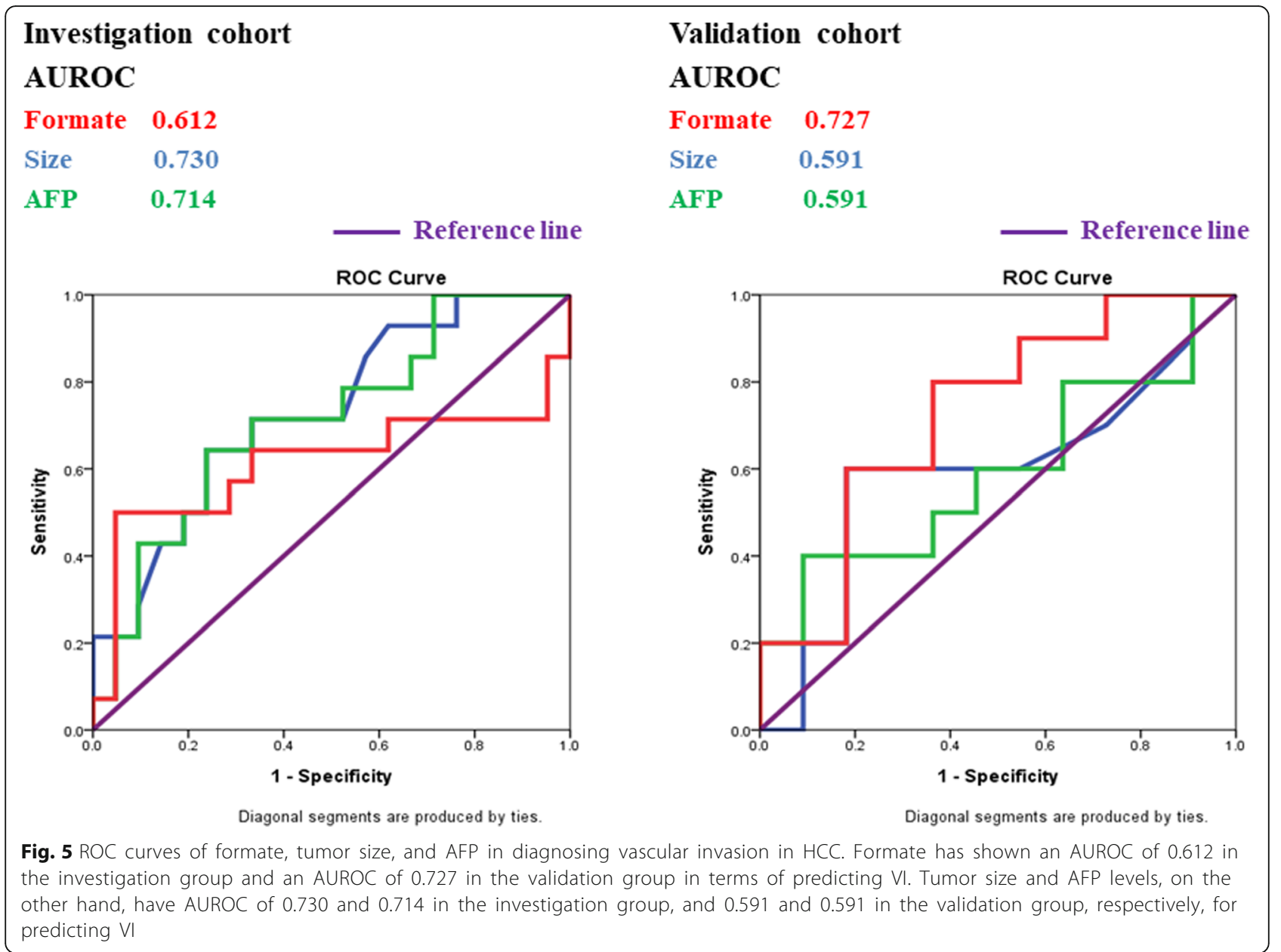

to lymphocyte ratio (dNLR), lymphocyte-to-monocyte ratio (LMR), age, and tumor size were independent predictors of micro-vascular invasion [30]. Kurokawa $\mathrm{T}$ et al. [31] discovered that next generation des-r-carboxy prothrombin (NX-DCP) may be used to predict the presence of VI in HCC. However, these researches are mostly correlation studies, and they failed to explain the mechanism underlying the association between the markers and VI. Moreover, most of them did not have validation cohorts to confirm their findings in the investigation cohort. As a result, it is of urgent need to develop a reliable biomarker to predict and explain VI prior to liver resection.

Several recent studies have demonstrated that formate is a useful biomarker in HCC [32-34]; however, what we have shown is that formate itself is a highly specific and selective biomarker for the prediction of VI and a combination of formate, tumor size and AFP further improves its performance. Our finding is supported by the study done by Meiser et al. that an increased formate overflow is a hallmark of oxidative cancers and that high formate levels promote tumor invasion [35]. Furthermore, they also discovered that inhibition of formate production by genetic interference could reduce cancer cell invasion. Their study is remarkable and further confirmed our finding that formate may be an effective serum marker to predict VI in HCC. Moreover, from the study mentioned above, the influence of formate is potentially targetable and may be utilized to develop treatment strategies against $\mathrm{HCC}$ with VI.

The results of the current study have shown that these metabolic changes are a result of carcinogenesis related to energy metabolism, lipid metabolism, and tricarboxylic acid (TCA) cycle. Given the small sample size and multiple comparisons, interpretation of the results must be cautious, even with validation data. As liver is a complex organ also responsible for lipid and amino acid metabolism, the use of high throughput liquid chromatography and mass spectrometry such as liquid chromatography mass spectrometry (LC-MS) with larger sample size is required. 


\section{Investigation cohort: combined formate+AFP+size AUROC 0.806}

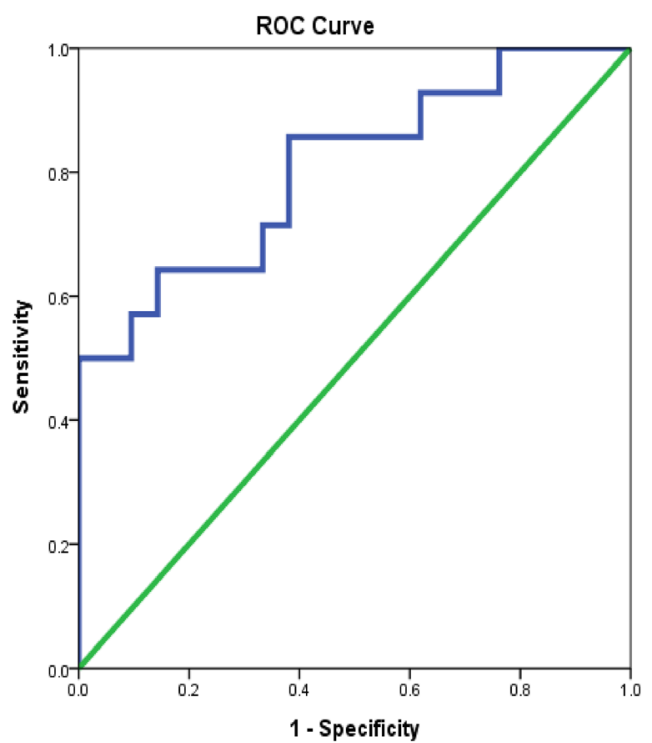

Validation cohort: : combined formate $+\mathrm{AFP}+$ size

AUROC 0.745

Fig. 6 Prediction of vascular invasion in HCC using a combination of formate, tumor size, and AFP. When combining formate, AFP, and tumor size, the AUROC in predicting microscopic VI raised to 0.806 in the investigation group and 0.745 in the validation group

\section{Conclusion}

In conclusion, metabolomic profiling can identify novel biomarkers that assist in the early diagnosis of AFPnegative HCC patients and recognition of microvascular invasion in order to facilitate preoperative surgical planning and postoperative follow-up. Further, larger scale prospective cohort studies are warranted to consolidate our findings.

\footnotetext{
Abbreviations

ACQ: Acquisition period; A-HCC: HCC with elevated or abnormal AFP levels (greater or equal to $15 \mathrm{ng} / \mathrm{mL}$ ); AFP: Alpha-fetoprotein; ALT: Alanine aminotransferase; AJCC: American Joint Committee on Cancer; ASPM: Abnormal spindle-like microcephaly associated; AST: Aspartate aminotransferase; AUC: Area under the curve; AUROC: Area under the curve of the receiver-operating characteristics; BMI: Body mass index; CA 199: Cancer antigen 19-9; CEA: Carcinoembryonic antigen; CGMH: Chang Gung Memorial Hospital; CGU: Chang Gung University; Cl: Confidence intervals; CT: Computed tomography; DM: Diabetes mellitus; ECOG: Eastern Cooperative Oncology Group; GGT: Gamma-glutamyltransferase; GCDCA: Glycochenodeoxycholic acid; HBV: Hepatitis B virus;

HCC: Hepatocellular carcinoma; HCV: Hepatitis C virus; ${ }^{1} \mathrm{H}-\mathrm{NMR}$ : Hydrogen-1 nuclear magnetic resonance; HR: Hazard ratios; ICG-15: Indocyanine green retention test at 15 min; INR: International normalized ratio; LC-MS: Liquid chromatography mass spectrometry; LNM: Lymph node metastasis; MRI: Magnetic resonance imaging; N-HCC: HCC with normal AFP levels (less than $15 \mathrm{ng} / \mathrm{mL}$ ); NX-DCP: Next generation des-r-carboxy prothrombin; OPLSDA: Orthogonal partial least squares-discriminant analysis; OS: Overall survival; RD: Relaxation delay; RFA: Radiofrequency ablation; ROC: Receiver-operating characteristics; TACE: Transarterial chemoembolization; TCA: Tricarboxylic acid; tm: Mixing time; TMS: Tetramethylsilane; TSP: 3-(trimethylsilyl)-
}

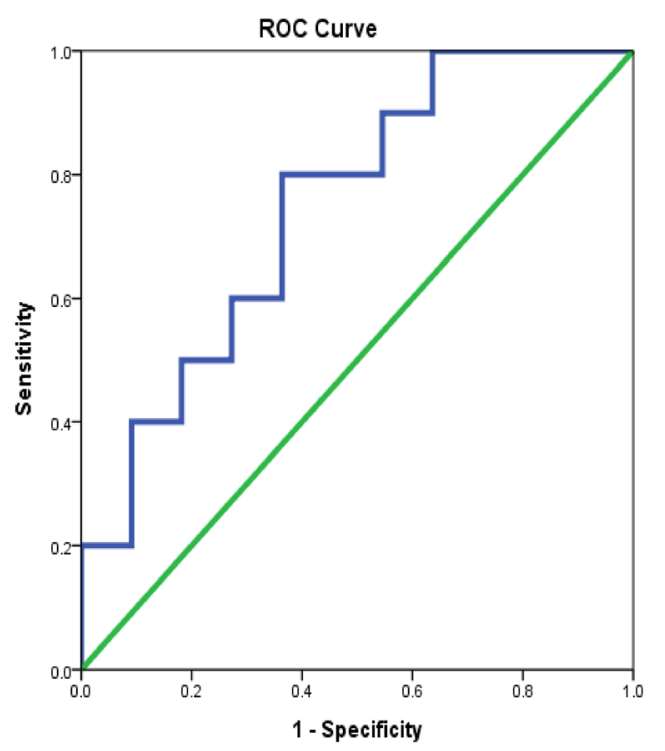




\section{Availability of data and materials}

All data generated or analyzed during the study are included in this published article. Raw data may be requested from the authors with the permission of the institution.

\section{Ethics approval and consent to participate}

This study was approved by the Institutional Review Boards (CGMH IRB No 201600940BOC501 and 201602025B0) of Chang Gung Memorial Hospital $(\mathrm{CGMH})$. Written informed consents were obtained from all subjects who entered the study.

\section{Consent for publication}

Not applicable

\section{Competing interests}

Hsin-I Tsai, Chao-Wei Lee, Jo-Chu Chiu, Meng-Han Chiang, Chang-Mu Sung, Yi-Chung Hsieh, Tony Kuo, Cheng-Yu Lin, Gi-gin Lin, and Ming-Chin Yu have no conflicts of interest or financial ties to disclose.

\section{Author details}

'Division of General Surgery, Department of Surgery, Linkou Chang Gung Memorial Hospital, Taoyuan, Taiwan. ${ }^{2}$ Graduate Institute of Clinical Medical Sciences, Chang Gung University, Taoyuan, Taiwan. ${ }^{3}$ College of Medicine, Chang Gung University, Taoyuan, Taiwan. ${ }^{4}$ Clinical Metabolomics Core Laboratory, Linkou Chang Gung Memorial Hospital, Taoyuan, Taiwan. ${ }^{5}$ Department of Medical Imaging and Intervention, Linkou Chang Gung Memorial Hospital, Taoyuan, Taiwan. ${ }^{6}$ Imaging Core Laboratory, Institute for Radiological Research, Linkou Chang Gung Memorial Hospital and Chang Gung University, Taoyuan, Taiwan. ${ }^{7}$ Department of Gastroenterology and Hepatology, Linkou Chang Gung Memorial Hospital, Taoyuan, Taiwan. ${ }^{8}$ Department of Anesthesiology, Linkou Chang Gung Memorial Hospital, Taoyuan, Taiwan.

Received: 10 March 2020 Accepted: 18 May 2020

Published online: 03 June 2020

\section{References}

1. Torre LA, Bray F, Siegel RL, Ferlay J, Lortet-Tieulent J, Jemal A. Global cancer statistics, 2012. CA: a cancer journal for clinicians. Mar 2015;65(2):87-108.

2. Department of Health ROC. Report of leading cancer-related death in 2014. Department of Health, Executive Yuan, Rebpulic of China. 2015.

3. Forner A, Llovet JM, Bruix J. Hepatocellular carcinoma. Lancet (London, England). Mar 31 2012;379(9822):1245-1255.

4. Tung-Ping Poon R, Fan ST, Wong J. Risk factors, prevention, and management of postoperative recurrence after resection of hepatocellular carcinoma. Ann Surg. Jul 2000;232(1):10-24.

5. Lee CW, Chan KM, Lee CF, et al. Hepatic resection for hepatocellular carcinoma with lymph node metastasis: clinicopathological analysis and survival outcome. Asian J. Apr 2011;34(2):53-62.

6. Shah SA, Cleary SP, Wei AC, et al. Recurrence after liver resection for hepatocellular carcinoma: risk factors, treatment, and outcomes. Surgery. Mar 2007;141(3):330-9.

7. Ibrahim S, Roychowdhury A, Hean TK. Risk factors for intrahepatic recurrence after hepatectomy for hepatocellular carcinoma. Am J Surg. Jul 2007;194(1):17-22.

8. Tandon P, Garcia-Tsao G. Prognostic indicators in hepatocellular carcinoma: a systematic review of 72 studies. Liver international : official journal of the International Association for the Study of the Liver. Apr 2009;29(4):502-10.

9. Lin SY, Pan HW, Liu SH, et al. ASPM is a novel marker for vascular invasion, early recurrence, and poor prognosis of hepatocellular carcinoma. Clinical cancer research : an official journal of the American Association for Cancer Research. Aug 1 2008;14(15):4814-4820.

10. Fu Y, Li J, Feng MX, et al. Cytohesin-3 is upregulated in hepatocellular carcinoma and contributes to tumor growth and vascular invasion. International journal of clinical and experimental pathology. 2014;7(5):212332.

11. Chen Y, Zhou J, Li J, Feng J, Chen Z, Wang X. Plasma metabolomic analysis of human hepatocellular carcinoma: diagnostic and therapeutic study. Oncotarget. Jun 172016.
12. Liu XN, Cui DN, Li YF, Liu YH, Liu G, Liu L. Multiple "Omics" data-based biomarker screening for hepatocellular carcinoma diagnosis. World J Gastroenterol. Aug 14 2019;25(30):4199-4212.

13. Di Poto C, Ferrarini A, Zhao $Y$, et al. Metabolomic characterization of hepatocellular carcinoma in patients with liver cirrhosis for biomarker discovery. Cancer epidemiology, biomarkers \& prevention : a publication of the American Association for Cancer Research, cosponsored by the American Society of Preventive Oncology. May 2017;26(5):675-83.

14. Luo $P$, Yin $P$, Hua $R$, et al. A large-scale, multicenter serum metabolite biomarker identification study for the early detection of hepatocellular carcinoma. Hepatology. Feb 2018;67(2):662-75.

15. Fitian Al, Cabrera R. Disease monitoring of hepatocellular carcinoma through metabolomics. World journal of hepatology. Jan 8 2017:9(1):1-17.

16. De Matteis S, Ragusa A, Marisi G, et al. Aberrant metabolism in hepatocellular carcinoma provides diagnostic and therapeutic opportunities. Oxidative medicine and cellular longevity. 2018;2018:7512159.

17. Ye G, Zhu B, Yao Z, et al. Analysis of urinary metabolic signatures of early hepatocellular carcinoma recurrence after surgical removal using gas chromatography-mass spectrometry. Journal of proteome research. Aug 3 2012;11(8):4361-4372.

18. Nahon P, Amathieu R, Triba MN, et al. Identification of serum proton NMR metabolomic fingerprints associated with hepatocellular carcinoma in patients with alcoholic cirrhosis. Clinical cancer research : an official journal of the American Association for Cancer Research. Dec 15 2012;18(24):6714-6722.

19. Zhou L, Liao Y, Yin P, et al. Metabolic profiling study of early and late recurrence of hepatocellular carcinoma based on liquid chromatographymass spectrometry. Journal of chromatography. B, Analytical technologies in the biomedical and life sciences. Sep 1 2014;966:163-170.

20. Beckonert $\mathrm{O}$, Keun HC, Ebbels TM, et al. Metabolic profiling, metabolomic and metabonomic procedures for NMR spectroscopy of urine, plasma, serum and tissue extracts. Nature protocols. 2007;2(11):2692-703.

21. Xia J, Psychogios N, Young N, Wishart DS. MetaboAnalyst: a web server for metabolomic data analysis and interpretation. Nucleic acids research. Jul 2009;37(Web Server issue):W652-W660.

22. Dan $Y$, Zhang $Y$, Cheng $L$, et al. Hepatitis $B$ virus $X$ protein $(H B X)$-induced abnormalities of nucleic acid metabolism revealed by (1)H-NMR-based metabonomics. Scientific reports. 2016;6:24430.

23. El-Serag HB, Rudolph KL. Hepatocellular carcinoma: epidemiology and molecular carcinogenesis. Gastroenterology. Jun 2007;132(7):2557-76.

24. Ferlay J, Shin HR, Bray F, Forman D, Mathers C, Parkin DM. Estimates of worldwide burden of cancer in 2008: GLOBOCAN 2008. International journal of cancer. Dec 15 2010;127(12):2893-2917.

25. Gao R, Cheng J, Fan C, et al. Serum metabolomics to identify the liver disease-specific biomarkers for the progression of hepatitis to hepatocellular carcinoma. Scientific reports. Dec 10 2015:5:18175.

26. Li H, Fan SF, Wang Y, Shen SG, Sun DX. Rapid detection of small molecule metabolites in serum of hepatocellular carcinoma patients using ultrafast liquid chromatography-ion trap-time of flight tandem mass spectrometry. Analytical sciences : the international journal of the Japan Society for Analytical Chemistry. 2017;33(5):573-8

27. Ladep NG, Dona AC, Lewis MR, et al. Discovery and validation of urinary metabotypes for the diagnosis of hepatocellular carcinoma in West Africans. Hepatology (Baltimore, Md.). Oct 2014:60(4):1291-1301.

28. Lu Y, Li N, Gao L, et al. Acetylcarnitine is a candidate diagnostic and prognostic biomarker of hepatocellular carcinoma. Cancer Res. May 15 2016; 76(10):2912-2920.

29. Chen C, Chen DP, Gu YY, et al. Vascular invasion in hepatitis B virus-related hepatocellular carcinoma with underlying cirrhosis: possible associations with ascites and hepatitis B viral factors? Tumour biology: the journal of the International Society for Oncodevelopmental Biology and Medicine. Aug 2015; 36(8):6255-63.

30. Li P, Huang W, Wang F, et al. Nomograms based on inflammatory biomarkers for predicting tumor grade and micro-vascular invasion in stage I/II hepatocellular carcinoma. Bioscience reports. Sep 252018.

31. Kurokawa T, Yamazaki S, Mitsuka Y, Moriguchi M, Sugitani M, Takayama T. Prediction of vascular invasion in hepatocellular carcinoma by nextgeneration des-r-carboxy prothrombin. Br J Cancer. Jan 12 2016;114(1):53-58.

32. Cox IJ, Aliev AE, Crossey MM, et al. Urinary nuclear magnetic resonance spectroscopy of a Bangladeshi cohort with hepatitis-B hepatocellular carcinoma: a biomarker corroboration study. World journal of gastroenterology. Apr 28 2016;22(16):4191-4200. 
33. Shariff MI, Kim JU, Ladep NG, et al. Urinary metabotyping of hepatocellular carcinoma in a UK cohort using proton nuclear magnetic resonance spectroscopy. Journal of clinical and experimental hepatology. Sep 2016;6(3): 186-94.

34. Shariff MI, Tognarelli JM, Lewis MR, et al. Plasma lipid profiling in a rat model of hepatocellular carcinoma: potential modulation through quinolone administration. Journal of clinical and experimental hepatology. Dec 2015;5(4):286-94.

35. Meiser J, Schuster A, Pietzke $M$, et al. Increased formate overflow is a hallmark of oxidative cancer. Nat Commun. Apr 10 2018;9(1):1368.

\section{Publisher's Note}

Springer Nature remains neutral with regard to jurisdictional claims in published maps and institutional affiliations.

Ready to submit your research? Choose BMC and benefit from:

- fast, convenient online submission

- thorough peer review by experienced researchers in your field

- rapid publication on acceptance

- support for research data, including large and complex data types

- gold Open Access which fosters wider collaboration and increased citations

- maximum visibility for your research: over $100 \mathrm{M}$ website views per year

At BMC, research is always in progress.

Learn more biomedcentral.com/submissions 